J. Urol. Urogynäkol. AT 2021 · 28:124-129 https://doi.org/10.1007/s41972-021-00148-4 Angenommen: 6. Oktober 2021 Online publiziert: 29. Oktober 2021 (c) Springer-Verlag GmbH Austria, ein Teil von Springer Nature 2021

\section{Alexander Piotrowski ${ }^{1} \cdot$ Fabian Siegel $^{2}$}

1 Urologie, Oberösterreichische Gesundheitsholding GmbH, PEK Klinikum Steyr, Steyr, Österreich ${ }^{2}$ Abteilung für Biomedizinische Informatik am Zentrum für Präventivmedizin und Digitale Gesundheit (CPD-BW), Medizinische Fakultät Mannheim der Universität Heidelberg, Universitätsmedizin Mannheim, Mannheim, Deutschland

\title{
Was können die Maschinen?
}

\section{Praktischer Nutzen von Big Data und Künstlicher Intelligenz in der Medizin}

\section{Einleitung}

Die Fähigkeiten der Computer entwickeln sich in einem rasanten Tempo und unterstützen uns zunehmend auch im Alltag. Große Schritte erzielten hierbei Big Data und die Künstliche Intelligenz (KI). Bei Big Data können durch die Analyse riesiger Datenmengen in kurzer Zeit noch unbekannte Zusammenhänge aufgedeckt und daraus neue Schlüsse gezogen werden. Diese Techniken werden auch verstärkt in der Medizin eingesetzt, sind jedoch nicht für alle Bereiche gleich gut geeignet.

- Welche Aspekte können die Maschinen gut bearbeiten?

- Welchen Vorteil bringen diese neuen Technologien?

- Wo können diese am sinnvollsten eingesetzt werden?

\section{Big Data - das Öl der Zukunft}

Der Begriff Big Data ist durch großvolumige Informationen mit großer Vielfalt und einer hohen Änderungsgeschwindigkeit charakterisiert [1]. Diese Daten können aus verschiedenen Quellen wie persönlichen Onlineaktivitäten, kommerziellen Verhaltensweisen oder aus persönlichen Sensordaten stammen [2]. Eine Big-Data-Sammlung weist somit 3 typische V-Charakteristiken auf: ein großes Datenvolumen („volume“), eine breite Vielfalt der Datenbeschaffenheit („variety“) und eine hohe Entstehungsgeschwindigkeit der Daten („velocity“). Diese 3 Charakteristiken beschreiben die Big-Data-Sammlung. Im Einzelnen betrachtet zeigt sich Folgendes:

- Volumen: Die große Menge an Daten liegt im Exabytebereich und hat nach oben keine Begrenzung. Die Prognosen zeigen, dass die Produktion der Daten weltweit stark zunehmen wird. Es werden in der Zukunft somit für Analysen immer größere Datenmengen zur Verfügung stehen.

— „Velocity“: In der Analyse von Big Data werden die Daten meist in einer hohen Geschwindigkeit erzeugt und auch in dieser verarbeitet. Hierbei ist es wichtig, mit diesen hohen Geschwindigkeiten umgehen zu können, um zeitnah auf relevante Ereignisse reagieren zu können.

- „Variety“: Eine weitere Besonderheit von Big Data ist die Heterogenität der Daten. Sie stammen meist aus den unterschiedlichsten Quellen und weisen somit eine stark unterschiedliche Struktur und ein inhomogenes Format auf.

Die Sammlung, Analyse und Anwendung dieser Big Data im Bereich der Medizin entwickelten sich in den letzten Jahren zu einem zunehmend wachsenden Gebiet. Dies umfasst etliche Gebiete und Definitionen.

Bei der Analyse muss auf eine gut strukturierte Weiterverarbeitung geachtet werden, um valide Schlussfolgerungen ableiten zu können. Hierbei umfasst die Analyse eine Integration der heterogenen Daten, eine Qualitätskontrolle und Korrelationen zwischen den Daten- banken. Die Erstellung von prädiktiven Modellen über Data-Mining-Techniken erfordert genaue Kenntnisse der Limitationen der gewonnenen Daten. Richtig angewandt können aus ihnen äußerst wertvolle Informationen gewonnen werden. Big Data wird somit zu Recht als das Öl der Zukunft bezeichnet.

\section{Bisherige Entwicklung von Big Data}

Allein das Volumen der medizinischen Daten im Gesundheitswesen steigt mit einer atemberaubenden Geschwindigkeit. So wurden im Jahre 2013153 Exabyte ( 1 Exabyte $=1$ Mrd. Gigabyte) Daten produziert und im Jahr 2020 waren es bereits 2314 Exabytes (• Abb. 1). Dies entspricht einer jährlichen Steigerung um 48 \% [3].

Zu berücksichtigen ist allerdings auch, dass mehr Daten nicht immer mehr Informationen bedeuten. Grafische Oberflächen und Bilddaten verursachen viel Datenvolumen, aber nicht immer mehr Informationen.

\section{Was ist KI}

Der Begriff künstliche Intelligenz (KI) wurde von einem amerikanischen Informatiker, John McCarthy, bereits im Jahre 1956 auf der „Dartmouth Conference“ eingeführt [5]. Die KI hat in den letzten Jahren v. a. durch die gleichzeitige Anwendung von Big Data an erheblichen Fähigkeiten hinzugewonnen.

Der Einsatz von KI und Big Data wird in der nahen Zukunft einen Arzt nicht ersetzen. Oder vielleicht doch? Auf jeden 


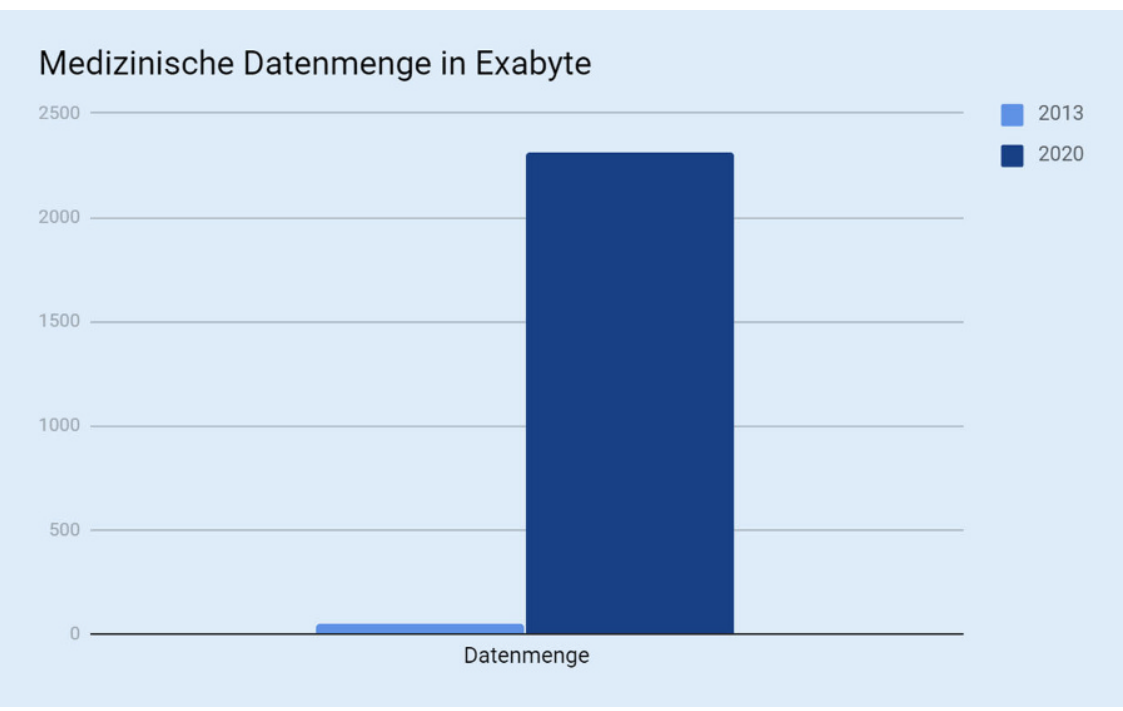

Abb. $1 \Delta$ Entwicklung der medizinischen Daten [4]

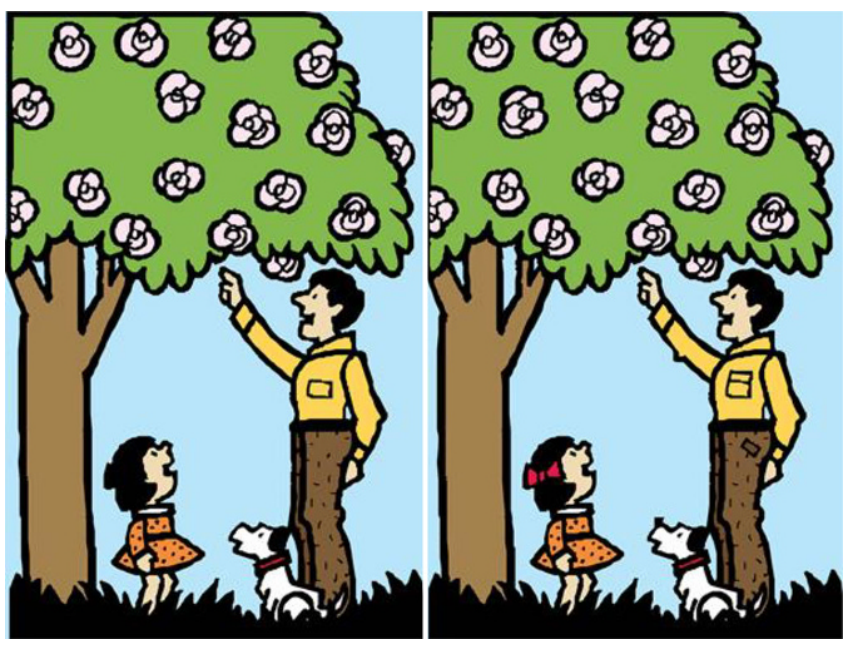

Abb. $2 \triangleleft$ Wie viele Fehler haben Sie gefunden? Wir Menschen mögen Suchbilder, weil wir darin nicht gut sind. Wie viele Millisekunden hätte die Maschine dafür gebraucht? (Bild aus pixabay.com)

Fall wird er ihn in bestimmten Bereichen ergänzen. Dieser Einsatz tritt v. a. in bestimmten Bereichen zuerst auf. Hierbei gibt es bestimmte Aspekte, bei denen die KI dem Menschen überlegen sind, da die Menschen nicht gut sind:

- im Quantifizieren,

- in komplexen Szenen die Änderungen festzustellen,

- Entscheidungen in mehreren Dimensionen zu treffen, sagt Prof. Dr.Ing. Horst K. Hahn vom Fraunhofer MEVIS in der DGU 2019 Hauptsitzung in Hamburg.

\section{Einsatz in der Analyse von Bilddaten}

Die Analyse von Bildern hat klassische statistische Verfahren bislang vor eine matisiert. Der Radiologe bzw. die Radiologin erhält die Ergebnisse der KI-Analyse unmittelbar am Beginn, wenn das Bild zur Befundung aufgerufen wird, und diese kann dann selbst entscheiden, ob die Informationen in den nachfolgenden diagnostischen Prozess einbezogen werden [6]. Die CT-Bilder können um 26\% schneller befundet [7] werden. In einer Untersuchung konnte hierdurch zudem eine deutliche Steigerung der Detektion von zuvor übersehener Lungenrundherde ermittelt werden [8].

Durch die KI kann zudem der gesamte Workflow in der Radiologie verbessert werden. Dies beginnt bei der Terminvergabe. Durch die Angabe von Knieschmerzen können bereits ein MRT mit der richtigen Spule gewählt und möglichst wenig Spulenumbauarbeiten im MRT zwischen den Patienten erreicht werden.

Durch die KI kann eine bessere Verlaufsbeurteilung erstellt werden. Für die Maschinen ist es viel leichter als für den Menschen, kleine Veränderungen in verschiedenen Bildern zu erkennen (- Abb. 2).

Der diesjährige 102. deutsche Röntgenkongress steht auch unter dem Motto „Intelligenz vernetzen“ und hat die künstliche Intelligenz als Schwerpunkt.

In der Urologie haben Bildbefundungssysteme mit KI eine lange Tradition, vom computerunterstützten Ultraschall auf der Suche nach Prostatakarzinomen [9] bis hin zur automatisierten Bewertung von Prostata-MRT-Bildern [10]. Hierdurch kann die Detektionsrate deutlich gesteigert werden [11].

Denkbar wäre in der Zukunft auch der Einsatz von KI in Krankenhäusern die, wie z. B. in der 3. Welt, keinen Radiologen zur Befundung zur Verfügung haben. Jedoch sprechen viele Gründe gegen den vollständigen Ersatz der Ärzte in der Befundung durch die künstliche Intelligenz [12]. Die endgültige Verantwortung für den Befund muss immer durch einen Menschen getragen werden. Doch nicht nur rechtliche Gründe verbieten einen alleinigen Einsatz der künstlichen Intelligenz. Künstliche Intelligenz ist begrenzt auf bekannte und trainierte Befundarten. Sie ist besonders gut in der Interpolation. Befinden sich jedoch Befunde außerhalb 
trainierter Grenzen und müssen extrapoliert werden oder finden sich Veränderungen, die im Trainingsdatensatz nicht auftraten und nicht klassifiziert wurden, so wird der Algorithmus diese auch nicht finden. Die Ergebnisse des Modells sind dann nicht vorhersehbar. Deshalb muss immer eine menschliche Plausibilitätsprüfung stattfinden.

\section{Einsatz in der Forschung}

Mit der zunehmenden Datenverarbeitungsmöglichkeit nehmen auch die Suche und Erschließung neuer Datenquellen zu. Als Datenquellen eigenen sich hervorragend die smarten Geräte. So liefern smarte Fieberthermometer und Wearables wie smarte Uhren oder Fitnesstracker große Mengen an analysierbaren Daten. Allein durch ein normales Mobiltelefon mit Ortungsfunktion können heute schon sehr präzise Daten für die medizinische Forschung gewonnen werden.

\section{Wearables}

Die telemedizinische Überwachung von Vitalzeichen und sonstiger Echtzeitgesundheitsdaten nimmt ebenso rapide $\mathrm{zu}$ wie die digitale Selbstmessung der Menschen über Wearables und Smartphones [13]. Dies und der Trend zum „quantified-self “ ist ein weiterer ungehobener Datenschatz.

Dieser Datenschatz wird aktuell v. a. durch führende Internetkonzerne genutzt. Aufgrund der engen Verzahnung mit Hersteller-Clouds besteht die Gefahr einer Konzentration solcher Daten bei einzelnen Herstellern. Glücklicherweise bieten die Betriebssysteme der Smartphones zunehmend Schnittstellen für den geordneten Export solcher Gesundheitsdaten.

\section{Genomsequenzierung}

Als äußerst erfolgreich haben sich die modernen Verfahren in der Genomsequenzierung bewährt. In Großbritannien werden durch das „100 000 Genomes Project" und in den Niederlanden durch "GoNL“ („Genome of the Netherlands") Genome entschlüsselt. Saudi-Arabien hat

J. Urol. Urogynäkol. AT 2021 · 28:124-129 https://doi.org/10.1007/s41972-021-00148-4

(c) Springer-Verlag GmbH Austria, ein Teil von Springer Nature 2021

\section{A. Piotrowski $\cdot$ F. Siegel \\ Was können die Maschinen? Praktischer Nutzen von Big Data und Künstlicher Intelligenz in der Medizin}

\section{Zusammenfassung}

Mit zunehmender Rechen- und Speicherkapazität hält in der Medizin fast unaufhaltsam die Anwendung von Big Data und künstlicher Intelligenz Einzug. Haupteinsatzgebiete sind bisher v. a. in der Radiologie und Pathologie, jedoch auch in weiteren Bereichen wie der Genomsequenzierung bis hin zur Psychiatrie zu finden. Durch die Auswertung von immer größeren Datenmengen wird nach Mustern gesucht, die die Arbeit der Ärzte erleichtern können, indem sie eine Vorselektion betreiben. Darüber hinaus bestehen auch Bemühungen bisher unerkannte Zusammenhänge aufzudecken. Große Datenmengen werden von den Patienten selbst über sog. Wearables erfasst. Wie können diese
Daten sinnvoll genutzt werden? Welche Gefahren bestehen? Auch in der Urologie gibt es Bestrebungen, eine Verbesserung der Behandlung von z. B. Harnwegsinfektionen oder Nierensteinen durch den Einsatz von vernetzten Computersystemen zu erreichen. Auf dem Gebiet von Big Data und Künstlicher Intelligenz sind in den nächsten Jahren große Fortschritte zu erwarten. Es wird auch zu einer erheblichen Erweiterung der Einsatzgebiete kommen, und wir sind somit erst am Anfang der Entwicklung.

Schlüsselwörter

Künstliche Intelligenz · Big Data · Maschinelles Lernen · Bildanalyse · Forschung

\section{What can machines do? Practical benefits of big data and artificial intelligence in medicine}

\section{Abstract}

With increasing computing and storage capacity, the application of big data and artificial intelligence (Al) is almost inexorably finding its way into medicine. The main areas of application so far have been in radiology and pathology, as well as in other areas such as genome sequencing and psychiatry. By evaluating ever larger amounts of data, patterns are sought that can make physicians' work easier by making a preselection. In addition, efforts are being made to uncover previously unrecognized connections. Large amounts of data are recorded by the patients themselves using so-called wearables. How can this data be used meaningfully? What are the dangers? Efforts are also being made in urology to improve the treatment of urinary tract infections and kidney stones, for example, through the use of networked computer systems. Great progress can be expected in the next few years in the field of big data and Al. An enormous expansion of the areas of application is going to come; as such, we are only at the beginning of the development.

\section{Keywords}

Artificial intelligence - Big data - Machine learning $\cdot$ Image analysis $\cdot$ Research das „Saudi Human Genom Programme“ begonnen, und in den USA hat Obama 2015 die „Precision Medicine Initiative“ gestartet, mit dem Ziel, genetische und medizinische Daten von 1.000.000 Personen zu sammeln [14].

Diese riesigen Datenmengen stellen einen so großen Datenpool dar, dass dieser mit herkömmlichen Methoden kaum zu bewältigen ist. Jedoch können durch die Nutzung von Big-Data-Techniken neue wissenschaftliche Entdeckungen und medizinische Erkenntnisse möglich werden.

\section{Medikamentenentwicklung}

Ein Durchbruch gelang Ende letzten Jahres der Google-Tochter Deep Mind. Diese kann mit KI die Proteinfaltung vorhersagen [15]. Die Vorhersage der Proteinstruktur ist für die Medikamentenentwicklung sehr wichtig. An diesem Problem haben sich Biochemie und Bioinformatik seit 50 Jahren die Zähne ausgebissen [16]. Durch die Lösung dieses Problems mit KI konnte ein Quantensprung in der Medikamentenentwicklung durch eine viel höhere Zielgenauigkeit mit we- 
niger Ausschuss erreicht werden. Dies trug auch maßgeblich zur mRNA-Impfstoffentwicklung bei.

\section{Erleichterung der Diagnostik}

Sogar in der Psychiatrie hat die KI Einzug gehalten. Eine Studie konnte hierbei beweisen, dass durch die Maschinen mit einer höheren Sensitivität, jedoch geringeren Spezifität diejenigen Patienten, die in einem Hochrisikokollektiv eine Psychose entwickeln werden, identifiziert werden konnten [17].

Auch in der Urologie tut sich einiges. So wurde in Deutschland 2016 die erste Phase der Medizininformatikinitiative gestartet, bei der sich nach Ausschreibungs- und Auswahlrunden 4 Konsortien bildeten, die Patientendaten für Therapie und Forschung verbundübergreifend verfügbar machen. Hierbei handelt es sich um Zusammenschlüsse mehrerer Universitätsklinika mit Hochschulen und Industriepartnern [18].

Im Rahmen des MIRACUM-Konsortiums wird aktuell hierdurch ein Harn- steinregister (RECUR) aufgebaut, um die Gründe für deren Entstehung endlich besser verstehen zu können.

In der Urologie werden die neuen Methoden auch eingesetzt. So kann künstliche Intelligenz eingesetzt werden, um für eine Harnwegsinfektion die passende antibiotische Therapie herauszusuchen [19]. Dies gelang dem System auch. Die zur Behandlung ausgewählten Antibiotika wiesen geringere Resistenzraten auf. Bei einer genaueren Betrachtung stellte man jedoch auch fest, dass es sich bei den vom Computer gewählten Antibiotika deutlich häufiger um Breitspektrumpräparate handelte. Hier sind weitere Entwicklungen notwendig.

\section{Einsatz in der Entschei- dungsfindung vor dem Arztbesuch}

Die KI wird auf der Basis von Big Data zunehmend für die Entscheidungsfindung in der Medizin vor dem Arztbesuch herangezogen werden. Studien, die den Erfolg ausreichend untersuchten, sind noch nicht genug vorhanden [20]. Jedoch zeigten Untersuchungen von Eligibility.com, dass $89 \%$ der Patienten in den USA ihre Beschwerden googeln, bevor sie die Hilfe eines Arztes erwägen [21]. Dies kann als ein „empowerment“ des Patienten angesehen werden, indem dieser stärker in die Behandlung mit einbezogen wird. Dieser Trend wird sicher noch weiter zunehmen und stellt eine sehr wichtige Entwicklung der Medizin der Zukunft dar.

Durch die Smartphone-App von Vocalis können inzwischen mit künstlicher Intelligenz auch chronisch-obstruktive Lungenerkrankungen im Anfangsstadium erkannt werden. Die Systeme wurden in der aktuellen Pandemie auch weiterentwickelt, um SARS-CoV-2-positive von -negativen Patienten zu unterscheiden. Dies kann auch in den normalen Alltag übertragen werden. Dies kann so weit gehen, dass in der Zukunft bei einem normalen Sprachkomando der Gesundheitsstatus analysiert und Ihr Roboter - Ihre Siri, Ihre Alexa - „Oh, Sie sind erkältet" sagen wird, behauptet Björn Schuller, ein Spezialist für Sprach-

Hier steht eine Anzeige. 
und Emotionserkennung der Universität Augsburg [22]. Dieser mahnt aber auch, dass die automatisierte Stimmanalyse noch ein neues Gebiet ist und eine Reihe möglicher Probleme bietet, die von fehlerhaften Diagnosen bis zum ungewollten Eindringen in die Privatsphäre der Patienten reicht.

\section{Probleme der neuen IT- Technologien}

Bei den neuen IT-Technologien gibt es jedoch auch Probleme, die angegangen werden müssen.

\section{„Garbage in - garbage out"}

Gerne wird künstliche Intelligenz als Allheilmittel für schlechte Datenqualität dargestellt. Wenn eine gute Datenerhebung nicht möglich oder zu teuer ist, wird gehofft, mittels großen Datenmengen und künstlicher Intelligenz dieses Defizit ausgleichen zu können.

\section{Nachvollziehbarkeit der Entscheidungen}

Ein Nachteil der künstlichen Intelligenz ist, dass die Entscheidungen des selbstlernenden und sich somit selbst trainierenden Systems nicht unbedingt nachvollzogen werden können. Es stellt somit eine Blackbox dar. Je gewichtiger der Einfluss der KI auf wichtige Entscheidungen in der Medizin, aber auch in anderen Bereichen wie der Polizei, den Banken usw. wird, haben Fehlentscheidungen der KI Konsequenzen [23]. Diesem Blackboxproblem der KI wird versucht, mit erklärbaren KI-Projekten entgegenzutreten [24].

Von den neuen technischen Möglichkeiten könnten sogar Gefahren ausgehen, die durch die Empfehlungen der künstlichen Intelligenz entstehen. So konnte in einem Testlauf ein medizinischer Chatbot mit KI dazu gebracht werden, einem Testpatienten zu empfehlen, sich zu töten [25].

\section{Datenschutz und KI}

Den europäischen Datenschutz bei einem Big-Data-Ansatz und KI zu gewähr- leisten, ist nicht ganz einfach, und dies führte auch zu einer Verlagerung von Projekten in außereuropäische Länder.

Dies wurde von der Politik erkannt [26]. Datenschutz kann ein Teil der Lösung in der europäischen Forschung sein und Europa eine Vorreiterrolle für eine vertrauenswürdige künstliche Intelligenz innehaben [27].

\section{Weitere Entwicklung von Big Data und KI}

\section{Weiterentwicklung der Diagnostik}

Die neuen Technologien können mit ihren Algorithmen immer besser lernen, Muster wie Ärzte zu erkennen. Im Gegensatz zu diesen benötigen diese Algorithmen jedoch tausende korrekte Beispiele, um so lernen zu können. Diese Beispiele müssen in einer sehr sauberen Form digital vorliegen, damit die Maschinen hieraus direkt die richtigen Schlüsse ableiten können.

Besonders gut eignen sich hierfür Informationen, die bereits in digitalisierter Form vorliegen, wenn sie vom Arzt untersucht werden. Dies können u. a. Hautbilder, Augenbilder, Elektrokardiogramme oder CT-Aufnahmen sein. Und liegen genug Daten vor, können die Maschinen ähnlich gute diagnostische Resultate wie Experten erzielen.

Die Ergebnisse der Maschinen liegen jedoch in einem Bruchteil von Sekunden vor. Zudem ist es möglich, diese Analyse nahezu überall mit geringen Kosten einzusetzen. Somit könnte es möglich sein, dass in der Radiologie in naher Zukunft die Diagnostik in sehr guter Qualität zu einem niedrigen Preis angeboten werden kann [28].

Die Entwicklung stellt erst den Anfang dar. Neuere Systeme werden im mehr Datenquellen zur Beurteilung aufnehmen. Dies kann dann auch Bereiche wie die Genomik, Proteomik und, als eine der schwierigen Datenquellen bei Ärzten, auch handschriftliche Aufzeichnungen umfassen.

Die Maschinen werden somit auch in der Zukunft die Ärzte unterstützen. Ist bei all dieser Weiterentwicklung ein Arzt nicht durch die modernen Algorithmen ersetzbar? Bei genauem Hinsehen erscheint es jedoch als unwahrscheinlich, dass dies in absehbarer Zeit geschieht. Die Maschinen werden in der Diagnostik auffällige Bereiche markieren und der Arzt sich durch diese Vorauswahl auf die genaue Interpretation dieser Befunde fokussieren können.

\section{Weiterentwicklung der Behandlung}

Der Einsatz von Big Data und KI kann helfen, zu bestimmen, welche Faktoren bei einer Behandlung zum besten Behandlungsergebnis für den Patienten führen werden. Denn unterschiedliche Patienten sprechen sehr verschieden auf die Medikation oder die Therapiepläne an. Durch den Einsatz der Maschinen soll es durch komplexe Analysen möglich sein, diejenigen Faktoren herauszufinden, welche die Therapie beeinflussen. Die dadurch gewonnenen Erkenntnisse helfen den Ärzten dann, den bestmöglichen Behandlungsplan zu entwerfen.

All dies stellt erst den Beginn der Entwicklung dar. Es werden immer mehr Daten in der Medizin digitalisiert und übertragbar werden. Umso mehr kann in diesen Big-Data-Banken die KI durch komplexe Analyseprozesse genutzt werden, um hierin signifikante Verbindungen zu erkennen. Mit dem Wissen über diese können die Entscheidungen, schneller, preiswerter und v. a. präziser werden.

\section{Korrespondenzadresse}

Prim. Dr. Alexander Piotrowski

Urologie, Oberösterreichische Gesundheitsholding $\mathrm{GmbH}$, PEK Klinikum Steyr

Sierningerstraße 170, 4400 Steyr, Österreich Alexander.Piotrowski@ooeg.at

\section{Einhaltung ethischer Richtlinien}

Interessenkonflikt. A. Piotrowski und F. Siegel geben an, dass kein Interessenkonflikt besteht.

Für diesen Beitrag wurden von den Autoren keine Studien an Menschen oder Tieren durchgeführt. Für die aufgeführten Studien gelten die jeweils dort angegebenen ethischen Richtlinien. 


\section{Literatur}

1. Laney D (2001) 3D data management: Controlling data volume, velocity, and variety. META Group. blogs.gartner.com/doug-laney/files/2012/01/ ad949-3D-Data-Management-Controlling-DataVolume-Velocityand-Variety.pdf. Zugegriffen: 8. März 2001

2. Nilsen W, Kumar S, Shar A et al (2012) Advancing the science of mHealth. JHealth Commun 17:5-10

3. Minor LB Stanford Medicine 2017 Health Trends Report. https://med.stanford.edu/ content/dam/sm/sm-news/documents/ StanfordMedicineHealthTrendsWhitePaper2017. pdf. Zugegriffen: 23. Juli 2020 (Erstellt: 23. Juli 2020)

4. Masterarbeit "Ökonomische Relevanz des Einsatzes von Big Data in der Krankheitsprävention" von Alexander Piotrowski, MHBA Studiengang, Friedrich-Alexander Universität Erlangen-Nürnberg.

5. https://www.computerweekly.com/de/definition/ Kuenstliche-Intelligenz-KI. Zugegriffen: 21. Okt. 2021

6. https://www.kma-online.de/aktuelles/medizintechnik detail/kuenstliche-intelligenz-gewinnt-anbedeutung-in-der-radiologie-a-44254. Zugegriffen:21.0kt. 2021

7. Freedman M, Lo B, Seibel J, BromleyE (2011) Improved detection of lung nodules with novel software that suppresses the rib and clavicle shadows on chest radiographs. Radiology 260:265-273

8. Lo SB, Freedman MT, Gillis LB, White CS, Mun SK (2018) JOURNAL CLUB:Computer-Aided Detection of Lung Nodules on CT With a Computerized Pulmonary Vessel Suppressed Function. AJR Am J Roentgenol 210(3):480-488. https://doi.org/ 10.2214/AJR.17.18718. Epub 2018 Jan 16. PMID: 29336601

9. Loch T, Leuschner I, Genberg C et al (2000) Weiterentwicklung des Transrektalen Ultraschalls: Artifizielle Neuronale Netzwerk-Analyse (ANNA) in der Erkennung und Stadieneinteilung des Prostatakarzinoms. Urologe A 39:341-347

10. Youn SY, Choi MH, Kim DH, Lee YJ, Huisman $\mathrm{H}$ Johnson E, Penzkofer T, Shabunin I, Winkel DJ Xing $P$, Szolar D, Grimm R, von Busch $H$, Son $Y_{\text {, }}$ Lou B, Kamen A (2021) Detection and PIRADS classification of focal lesions in prostate MRI: Performance comparison between a deep learning-based algorithm (DLA) and radiologists with various levels of experience. Eur J Radiol 142:109894. https://doi.org/10.1016/j.ejrad.2021. 109894

11. Tokas T, Grabski B, Paul U, Bäurle L, Loch $T$ (2018) A 12-year follow-up of ANNA/C-TRUS image-targeted biopsies in patients suspicious for prostate cancer. World J Urol 36(5):699-704. https://doi.org/10.1007/s00345-017-2160-z

12. https://towardsdatascience.com/why-ai-willnot-replace-radiologists-c7736f2c7d80. Zugegriffen:21.0kt. 2021

13. Health Communication Network Pty Limited. https://www.medicaldirector.com/news/ future-of-health/2019/02/new-report-revealsthe-future-of-wearable-devices-in-healthcare. Zugegriffen:21.0kt. 2021

14. https://allofus.nih.gov/.Zugegriffen:21. Okt. 202

15. Callaway E (2020) 'It will change everything': DeepMind's Al makes gigantic leap in solving protein structures. Nature 588(7837):203-204. https://doi.org/10.1038/d41586-020-03348-4

16. https://deepmind.com/blog/article/alphafold-asolution-to-a-50-year-old-grand-challenge-inbiology.Zugegriffen:21.Okt. 2021
17. Koutsouleris N, Dwyer DB, Degenhardt F et al (2021) Multimodal machine learning workflows for prediction of psychosis in patients with clinical high-risk syndrome and recent-onset depression. JAMA Psychiatry 78:195-209 (https://jamanetwork.com/journals/ jamapsychiatry/fullarticle/2773732.)

18. https://www.medizininformatik-initiative.de/de/ ueber-die-initiative/phasen. Zugegriffen: 21. Okt. 2021

19. Yelin I, Snitser O, Novich G, Katz R, Tal O, Parizade M, Chodick G, Koren G, Shalev V Kishony R (2019) Personal clinical history predicts antibiotic resistance of urinary tract infections. $\mathrm{Nat}$ Med 25(7):1143-1152. https://doi.org/10.1038/ s41591-019-0503-6

20. Nagendran $M$, Chen $Y$, Lovejoy CA, Gordon AC, Komorowski M, Harvey H, Topol EJ, loannidis JPA, Collins GS, Maruthappu M (2020) Artificial intelligence versus clinicians: systematic review of design, reporting standards, and claims of deep learning studies. BMJ 368:m689. https://doi.org/ 10.1136/bmj.m689

21. The most Googled medical symptoms by state https://eligibility.com/medicare/states-mostgoogled-medical-symptom. Zugegriffen: 30 . Juli 2019

22. Anthes E (2020) Alexa, do I have COVID-19? Nature 586(7827):22-25. https://doi.org/10.1038/ d41586-020-02732-4

23. https://www.thinkautomation.com/bots-and-ai/ the-ai-black-box-problem/. Zugegriffen: 21. Okt. 2021

24. https://www.thinkautomation.com/eli5/eli5explainable-ai/.Zugegriffen:21.0kt. 2021

25. https://artificialintelligence-news.com/2020/10/ 28/medical-chatbot-openai-gpt3-patient-killthemselves/.Zugegriffen:21.Okt. 2021

26. Deutscher Bundestag - Im Spannungsfeld zwischen Datenschutz und Künstlicher Intelligenz. https://www.bundestag.de/dokumente/ textarchiv/2020/kw03-pa-enquete-kuenstlicheintelligenz-673918.Zugegriffen:21. März2021

27. https://ec.europa.eu/germany/news/20200219digitale zukunft-europas-eu-kommission-stelltstrategien-fuer-daten-und-kuenstlicheintelligenz_de.Zugegriffen:21.0kt. 2021

28. https://www.datarevenue.com/de-blog/ kuenstliche-intelligenz-in-der-medizin. Zugegriffen:21.0kt. 2021

Hinweis des Verlags. Der Verlag bleibt in Hinblick auf geografische Zuordnungen und Gebietsbezeichnungen in veröffentlichten Karten und Institutsadressen neutral.

\section{Wie sich Corona auf sexuell übertragbare Infektionen auswirkt}

Im letzten Jahr ist die Zahl der gemeldeten Infektionen mit Hepatitis $\mathrm{C}$ und HIV deutlich gesunken. Das Robert-Koch-Institut äußert beim Hauptstadtkongress 2021 die Sorge, dass sich die Zahl der unbewusst infizierten Personen vermehrt.

Insgesamt wurden während der globalen Corona-Pandemie $20 \%$ weniger HIVErstdiagnosen im Vergleich zum Vorjahr gemeldet. Gleichzeitig ist auch die Nachfrage nach dem Medikament, mit dem sich eine HIV-Infektion vorbeugen lässt, Präexpositionsprophylaxe (PrEP), gesunken. Ähnliche Entwicklungen lassen sich be den gemeldeten Fällen von Hepatitis C beobachten, die 2020 einen klaren Rückgang aufzeigten. Das RKI geht davon aus, dass die Situation in diesem Fall vermutlich vor allem auf die Unterdiagnose und Untererfassung von Gruppen wie Drogenkonsumenten, Häftlingen und Migranten zurückzuführen sei.

Die HIV-Erstdiagnosen könnten einerseits aufgrund der reduzierten Treffen mit Sexualpartnern und einer insgesamt geringeren Mobilität zurückgegangen sein, doch man müsse bedenken, dass gleichzeitig auch Angebot und Nachfrage von HIV-Tests gesunken seien. Dies könnte dazu führen, dass in Deutschland die Anzahl von HIV-Infizierten steigt, die nichts von ihrer Infektion wissen.

In diesem Zusammenhang wurde auf dem Hauptstadtkongress daran erinnert, dass Deutschland die Internationalen Ziele der UNAIDS im Jahr 2020 verfehlt hatte. Denn statt den angestrebten mindestens $90 \%$ haben nur rund $88 \%$ der HIV-Infizierten eine Diagnose, was bedeutet, dass eine HIV-Infektion immer noch zu oft übersehen wird. Das steigert schlussendlich die Gefahr, dass die Gruppe der unbewusst HIV-positiven Menschen wächst und diese unbewusst weitere Personen infizieren.

Quelle: Ärzte Zeitung (www.aerztezeitung.de) 